This item was submitted to Loughborough's Research Repository by the author.

Items in Figshare are protected by copyright, with all rights reserved, unless otherwise indicated.

\title{
Antennas on quasi synthetic media
}

PLEASE CITE THE PUBLISHED VERSION

http://dx.doi.org/10.1007/s00339-013-8026-3

\section{PUBLISHER}

(C) Springer

\section{VERSION}

AM (Accepted Manuscript)

\section{PUBLISHER STATEMENT}

This work is made available according to the conditions of the Creative Commons Attribution-NonCommercialNoDerivatives 4.0 International (CC BY-NC-ND 4.0) licence. Full details of this licence are available at: https://creativecommons.org/licenses/by-nc-nd/4.0/

\section{LICENCE}

CC BY-NC-ND 4.0

\section{REPOSITORY RECORD}

Njoku, Chinwe C., W.G. Whittow, and J.C. Vardaxoglou. 2019. "Antennas on Quasi Synthetic Media”. figshare. https://hdl.handle.net/2134/17928. 


\title{
Antennas on Quasi Synthetic Media
}

\author{
Chinwe C. Njoku1, William G. Whittow ${ }^{1}$, (J) Yiannis C. Vardaxoglou ${ }^{1 *}$ \\ ${ }^{1}$ School of Electronic, Electrical \& Systems Engineering, Loughborough University, Loughborough, LE11 3TU, UK \\ * E-mail: J.C.Vardaxoglou@lboro.ac.uk
}

\begin{abstract}
This paper examines the differences and/or similarities between some of the commonly used canonical equations describing the effective permittivity and permeability of heterogeneous substrates with dielectric or metallic inclusions, at microwave frequencies. Graphical results are presented for the different structures considered. Simulated results from heterogeneous mixtures with both dielectric and metallic inclusions are presented, and compared with results from the canonical equation representation. Simulated results of a patch antenna on a heterogeneous medium and on its homogenous equivalent have also been presented.
\end{abstract}

\section{Introduction}

The overall goal of this research is to one day, be able to apply nanomaterial fabrication techniques to the production of microwave antenna systems, such that these systems are made in one process. As such, an important aspect is to understand the electromagnetic (EM) properties of heterogeneous structures which are structures consisting of small-scale inclusions in a homogenous host. Heterogeneous structures have been of interest to engineers and physicists alike as they provide additional degrees of freedom when designing various electromagnetic structures. These heterogeneous structures can be applied to the design of microwave antennas to control the dielectric and possibly the magnetic properties, thus allowing the creation of novel substrates with pre-determined characteristics. EM advantages related to bandwidth, efficiency and size may be achieved as a by-product of being able to control the material's EM properties. In previous work, it has been shown that a larger bandwidth can be achieved with an as yet unrealizable substrate with equal permittivity and permeability [1]. In addition, using textured dielectrics in which the permittivity of the substrate is mapped to the electric field intensity, such as with a patch antenna, has been shown to improve antenna performance [2-5]. Fig. 1 shows an example of how these inclusions can be smoothly varied in a substrate to dictate the local effective permittivity.

Lord Rayleigh in [6] was the first reported person to examine these structures in the mid- $19^{\text {th }}$ century but more commonly used is the paper by Lewin in [7]. Since his analysis in 1946, extensions, modifications and alternatives to his equations for the effective permittivity, $\varepsilon_{\text {eff }}$ and effective permeability, $\mu_{\text {eff }}$ of a semi-infinite heterogeneous structure with spherical inclusions have been published [813]. An initial analysis carried out by the authors in [14] in which these different canonical equations for the $\varepsilon_{\text {eff }}$ and $\mu_{e f f}$ are listed and briefly explained, is being extended in this paper. These equations are compared and analysed in further detail here.

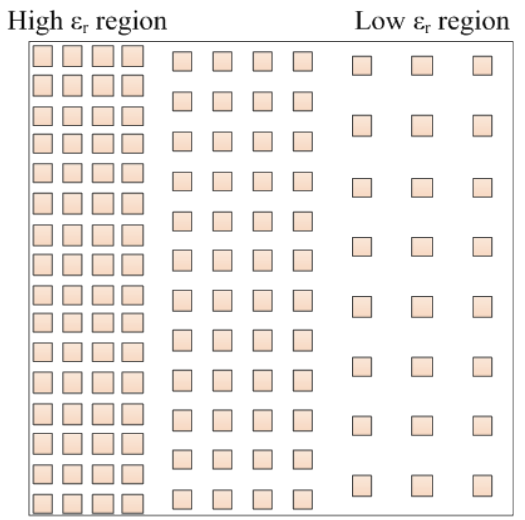

Figure 1: A sketch showing how the permittivity can be varied within the substrate by changing the spacing of the inclusions (not to scale)

Section 2 highlights the differences and similarities between the different effective medium theories in order to determine which of these theories are robust enough to cover the structures investigated in this research, and which ones were not suitable. Mathematical manipulations and application of assumptions/conditions such as using metallic inclusions and using uniform inclusion spacing in all three axes, were carried out on some of the equations to show their similarities. Section 3 presents some graphical results using these equations while Section 4 investigates a heterogeneous structure with both dielectric and metallic inclusions and presents simulated results of different configurations. The complete simulation and inversion process of the scattering (S-) parameters has been described in detail and used in [15], [16]. A patch antenna on a heterogeneous substrate with dielectric cubic inclusions and on the homogenous equivalent substrate, have been simulated and the return loss values presented in Section 5. Finally, conclusions are given in Section 6. The values of $\varepsilon$ and $\mu$ given here are absolute values (except otherwise stated) and relative to those of free space, $\varepsilon_{o}$ and $\mu_{o}$. 


\section{Comparative Analysis}

\subsection{Mathematical Comparisons}

By assuming conducting spheres in a cubic array and using algebraic manipulations, it is shown below that the equations from [7] and [10] for the $\varepsilon_{\text {eff }}$ and $\mu_{e f f}$ of these heterogeneous structures are similar to each other. These equations are given respectively in equations (1) and (2).

$$
\begin{aligned}
& \varepsilon_{e f f}=\varepsilon_{1}\left(1+3 p /\left(\frac{\varepsilon_{p}+2 \varepsilon_{1}}{\varepsilon_{p}-\varepsilon_{1}}-p\right)\right) ; \\
& \mu_{e f f}=\mu_{1}\left(1+3 p /\left(\frac{\mu_{p}+2 \mu_{1}}{\mu_{p}-\mu_{1}}-p\right)\right)
\end{aligned}
$$

where $\left(\varepsilon_{1}, \mu_{1}\right)$ are the $\varepsilon$ and $\mu$ of the host medium; $p=$ $\frac{4}{3} \pi a^{3} / s^{3}$ is the inclusions' volume ratio; $(a, s)$ are the radius and centre-to-centre spacing of the inclusions; $\left(\varepsilon_{p}, \mu_{p}\right)$ are the effective $\varepsilon$ and $\mu$ of the inclusions, given by:

$$
\frac{\varepsilon_{p}}{\varepsilon_{2}}=\frac{\mu_{p}}{\mu_{2}}=\frac{2(\sin \theta-\theta \cos \theta)}{\left(\theta^{2}-1\right) \sin \theta+\theta \cos \theta}=F(\theta)
$$

where the constant, $\theta=k a \sqrt{\mu_{2} \varepsilon_{2}} ; k=2 \pi / \lambda$ and $\lambda=$ operating wavelength; $\left(\varepsilon_{2}, \mu_{2}\right)$ are the bulk $\varepsilon$ and $\mu$ of the inclusion material.

$$
\varepsilon_{e f f}=1+\frac{N \alpha_{1}}{1-\alpha_{1} C} \quad \mu_{e f f}=1+\frac{N \beta_{1}}{1-\beta_{1} C}
$$

where $\alpha_{1}, \beta_{1}$ are the inclusion's electric and magnetic polarisabilities, $N$ is the density of the inclusions and $C$ is the interaction constant.

The electric and magnetic polarisabilities for spherical inclusions are given by: $\alpha_{1}=4 \pi a^{3}$ and $\beta_{1}=-2 \pi a^{3}$ [10] respectively. Assuming uniform spacing, $s$ in the $x, y$, and $z$ directions,

$$
C=\frac{1}{\pi s^{3}}\left[1.202-16 \pi^{2} K_{0}(2 \pi)\right]
$$

where $K_{0}(\cdot)$ is the modified Bessel function of the second kind and reduces to $C=1.06 / \pi s^{3}$ using the approximation for the Bessel function for large variables. Substituting this into equation (2) gives,

$$
\varepsilon_{e f f}=\frac{1+8.33 a^{3} / s^{3}}{1-4.24 a^{3} / s^{3}} \quad \mu_{e f f}=\frac{1-4.16 a^{3} / s^{3}}{1+2.12 a^{3} / s^{3}}
$$

As discussed in [7], for metal particles, $\varepsilon_{p}$ is much greater than $\varepsilon_{1}$. Thus the expression for permittivity in equation (1) reduces to

$$
\varepsilon_{\text {eff }}=\varepsilon_{1}[1+3 p /(1-p)]
$$

Substituting the expression for $p=4 \pi a^{3} / s^{3}$ reduces the expression in the square brackets above to $\frac{1+8.38 a^{3} / s^{3}}{1-4.19 a^{3} / s^{3}}$, which is very similar $( \pm 0.05$ difference in the multipliers of $a^{3} / s^{3}$ ) to the expression for $\varepsilon_{e f f}$ in equation (3). Since a "lightweight binder" like Polyfoam which has a dielectric constant of 1.05 [17] is used in the analysis in [10], $\varepsilon_{1}$ can be approximated to $\varepsilon_{0}$.

Also, using the approximation for $\mu_{e f f}$, if the particles are non-magnetic such that $\mu_{p} \rightarrow 0$, the expression for permeability in equation (2) is reduced to

$$
\mu_{\text {eff }}=\mu_{1}(1-3 p / 2+p)
$$

Substituting for $p$, reduces the expression in the bracket above to $\frac{1-4.19 a^{3} / s^{3}}{1+2.09 a^{3} / s^{3}}$, which is again similar to (3). Since the binder material is non-magnetic, $\mu_{1} \approx \mu_{0}$.

From the foregoing calculations, the expressions for the $\varepsilon_{e f f}$ and $\mu_{\text {eff }}$ of a dielectric host containing spherical particles, obtained in [7] and [10] are almost the same assuming: uniform simple cubic spacing, non-magnetic spherical conducting inclusions and a host permittivity close to that of air, $\varepsilon_{0}=1$.

Furthermore, the expressions for the effective parameters by Collin [10] as shown in equation (2) are similar to those of Cai et al [8] as shown in equation (5).

$$
\varepsilon_{\text {eff }}=1+\frac{N \alpha_{1}}{1-N \alpha_{1} / 3} \quad \mu_{\text {eff }}=1+\frac{N \beta_{1}}{1-N \beta_{1} / 3}
$$

In [10], the interaction constant for equally-spaced spheres was obtained as $C=1.06 / \pi s^{3}$. In this case, $N=s^{-3}$, and therefore $C=N \times 1.06 / \pi$. Since $\frac{1.06}{\pi} \cong 1 / 3$, substituting in equation (2) yields $\varepsilon_{e f f}=1+\frac{N \alpha_{1}}{1-N \alpha_{1} / 3}$, which is the same as Cai's equation in [8]. The equations in [10] also suggest that they can be applied to mixtures with non-uniform spacings along the $x, y$ and $z$ axes.

Although [8-10], [12] have used polarisabilities in their determination of effective constitutive parameters, they have represented these expressions differently. As an example, the expression for polarisability in equation (4) [8] and that in [12] yield very similar graphs as shown in Fig. 2 when used to plot the $\varepsilon_{\text {eff }}$ against frequency, even though they are mathematically not exactly equal. In addition, although there is a ' $4 \pi$ ' product difference in their equations for the effective properties appearing in the numerator and denominator as in [12], the $4 \pi$ 's cancel each other out. Another disparity is in the expressions for polarisability in [8] and [12] which is due to the presence of the host medium's refractive index value in the expression in [12].

\subsection{General Comparisons}

The limit of the validity of formulas is that the spherical particles should not be densely packed. This is similar to that in [10] whose validity is based on the relationship between the obstacles' dimension and their spacing. Notable is the difference in the representation of the Clausius-Mossotti equation by Sihvola [9] from that used in equation (5) by Cai et al [8], by the presence of the ' $\varepsilon_{1}$ ' term in the denominator. This is because equation (5) by Cai et al from [8] takes into account the EM properties of the inclusions but not that of the host or assumes the host has EM properties close to that of free space.

The difference between the Maxwell-Garnett equation and Lewin's formula from [7] is that while Lewin makes the particles exhibit an effective $\varepsilon$ and $\mu$ due to their small size, Maxwell-Garnett assumes that the smaller size particles have the same EM properties as when in their bulk forms. As the value of $\theta$ (see equation (1b)) changes from being very small to being large and complex as the size-towavelength ratio increases, this variation in permittivity 
decreases. The value of the expression for $\theta$ in [7], [13], [18] differs with the size parameter, $x\left(=k a \sqrt{\varepsilon_{1} \mu_{1}}\right)$ in [8], [12], in that the effect of $\varepsilon_{2}$ and $\mu_{2}$ are not accounted for. $\sqrt{\varepsilon_{2} \mu_{2}}$ in the expression for $\theta$ is equal to the refractive index of the material of the particle, say $n_{2}$, while $x$ uses the refractive index of the host medium, $n_{0}$.

Kolmakov et al in [13] adopts the formula by Sihvola in [9] for mixtures with more than one size of spherical inclusions to write an expression for $\varepsilon_{\text {eff }}$ and $\mu_{\text {eff }}$ of a heterogeneous medium with a single-size sphere but having two different frequency-dependent polarisabilities. It takes into account the fact that the radii, the polarisabilities, the volume fractions and volume densities of the spheres will vary in magnetic and electric resonance modes. This is the primary difference from Lewin's study in [7] where these variations are not taken into account. The spheres have different radii and were arranged accordingly. In their study the particle spacing was also taken to be much smaller than the wavelength - in line with the conditions for formulae validity in [7]. Numerical simulations were carried out on dielectric spherical and cylindrical particle arrays and it was found out that when the inclusions' spacing was close to a wavelength, or even approximately half of the wavelength, the effective medium approach is not best suited for such mixtures [13]. This is because "the particle interferes with the normal behaviour" of the host in [7].

Other minor differences are that: the difference in the formulas for effective constitutive parameters in [7], [10] and [11] can be attributed to the product term difference of $\varepsilon_{o}$ in their expressions for dipole moment, $\overline{\boldsymbol{p}}$.

\section{Graphical Results}

In order to further highlight the similarities and differences between these equations, a variation of the $\varepsilon_{\text {eff }}$ and $\mu_{\text {eff }}$ with particle size are given in Fig. 2. Data used: frequency, $f=3 \mathrm{GHz}$, spacing, $s=200 \mu \mathrm{m}$, inclusion radius, $a=1$ $100 \mu \mathrm{m}$, the dielectric host medium, $\varepsilon_{1}=2.25(\tan \delta=$ $0.001)$. The inclusions are made of Copper $(\sigma=5.8 \times$ $10^{7} \mathrm{~S} / \mathrm{m}$ ); the relative permittivity of Copper was obtained as $\varepsilon_{2}=(1.2-j 103.5) \times 10^{6}$, from first principles using the Drude model [19], $\mu_{1}=\mu_{2}=1$.

The results from [8] and [10] agree with each other but do not agree with those from [7], [9], [12], [13] as [8] and [10] do not include the value of the host permittivity, $\varepsilon_{1}$ in their equations and so will only agree with the others when the host permittivity, $\varepsilon_{1} \cong 1$. Even though $\mu_{1}=\mu_{2}=1$, $\mu_{\text {eff }}$ reduces with increase in the inclusions' volume fraction because the inclusions are metallic and may create diamagnetism with the medium when illuminated by an external magnetic field. This diamagnetic effect has been reported in previous work [20]. But due to the denominator term, $\mu_{2}-\mu_{1}$ being equal to 0 in [9], this results in its $\mu_{\text {eff }}=1$.

Formulations from [9], [10] do not have frequency terms, therefore only equations from [7], [8], [12], [13] are used to understand the effect of variation of the operating frequency with the EM properties of the heterogeneous mixture as shown in Fig. 3. Here, it is assumed that the permittivities of the host and the inclusions are constant with frequency. Data used: $1-30 \mathrm{GHz} ; a=100 \mu \mathrm{m}, s=220 \mu \mathrm{m}, \mu_{1}=\mu_{2}=1$, $\varepsilon_{1}=2.25(\tan \delta=0.001)$ and $\varepsilon_{2}=(1.2-j 103.5) \times 10^{6}$.

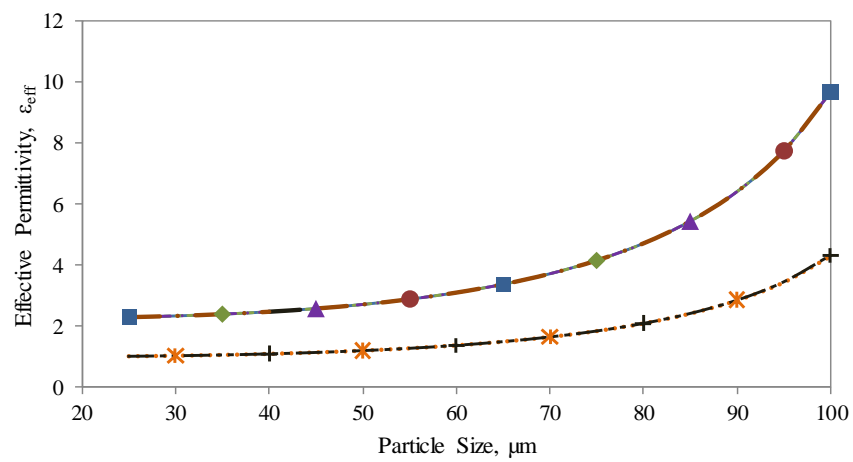

(a)

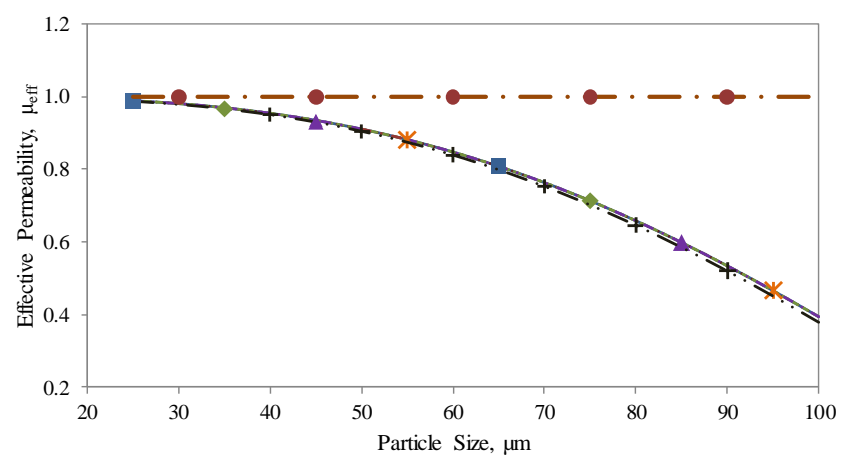

(b)

Figure 2: Variation of (a) $\varepsilon_{e f f}$ and (b) $\mu_{\text {eff }}$ with spherical

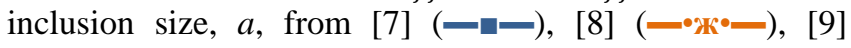
$(=\bullet=\bullet \bullet=\bullet=),[10](-\bullet \cdot \bullet \bullet-),[12](--\bullet-)$ and [13] $(" \Delta-")$.

As shown in Fig. 3, $\varepsilon_{\text {eff }}$ from [8] differs significantly from the others because $\varepsilon_{1}>1$ which is not included in its equation for $\varepsilon_{\text {eff }}$. The other results agree over the frequency range considered. For the case of $\mu_{e f f}$, all the results from the equations agree with each other. As the frequency increases an initial reduction in the $\mu_{\text {eff }}$ from 1 is seen, and then $\mu_{\text {eff }}$ remains nearly constant at approximately 0.5 . This may be due to the rather high value of $\varepsilon_{2}$ for metallic inclusions.

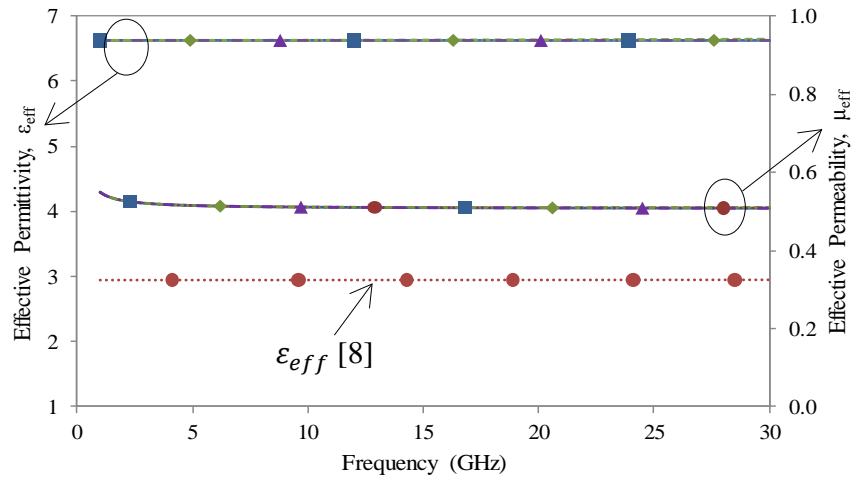

Figure 3: Variation of $\varepsilon_{e f f}$ and $\mu_{\text {eff }}$ with frequency from [7]

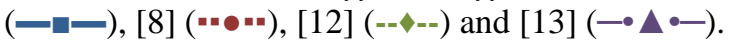




\section{Mixed Inclusions}

More advanced studies are carried out in [9], [21] for mixtures that include two or more homogenous material particles of equal or different sizes. This can also allow the creation of anisotropic materials. Here, the effective permittivity is a function of summation terms where the volume fractions of each included material and their respective permittivities are examined separately. The different arrangements of these structures examined in this section are shown in Fig. 4. In this section, analytical results are compared with FDTD simulations in conjunction with a retrieval algorithm for the $\varepsilon_{e f f}$ and $\mu_{\text {eff }}$.

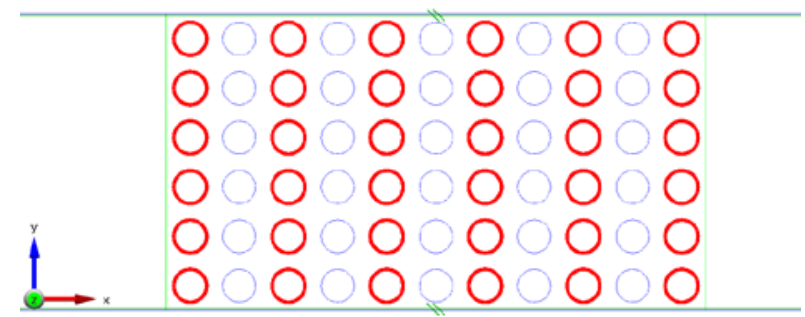

(a)

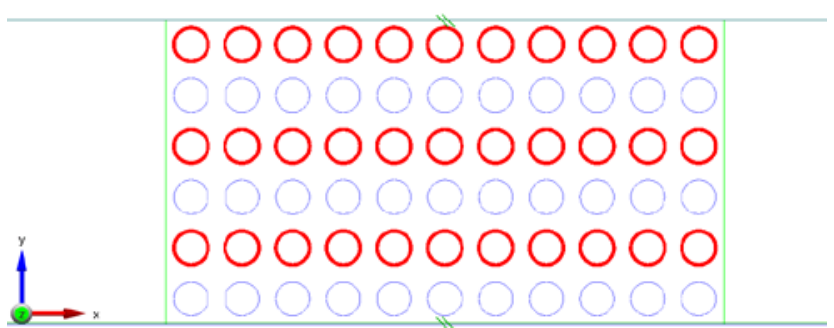

(b)

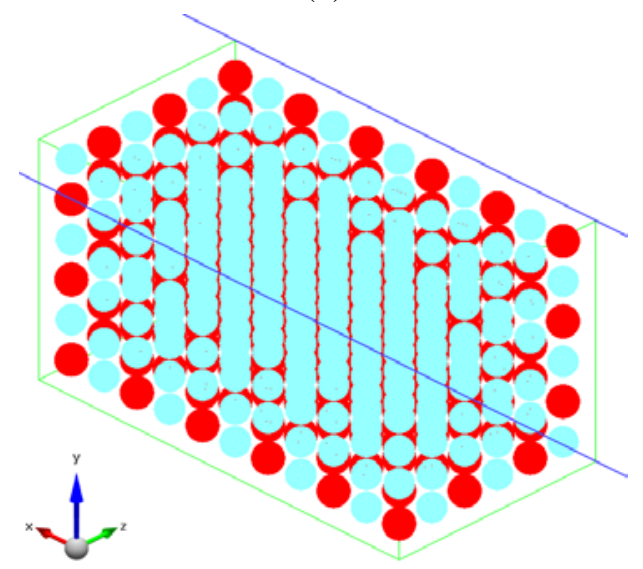

(c)

Figure 4: (a) $y$-oriented, (b) $x$-oriented and (c) alternating arrays of metallic (red, thick circles) and dielectric (blue, thin circles) inclusions in a homogenous host. The orientations refer to the orientations of the columns/rows of the metallic inclusions shown in bold red lines in (a) and (b).

Using the inversion process as described in [15] for extracting the $\varepsilon_{e f f}$ and $\mu_{\text {eff }}$ of heterogeneous structures, the results shown in Fig. 5 were obtained. Data used: $f=1$-20 $\mathrm{GHz}, a=100 \mu \mathrm{m}, s=300 \mu \mathrm{m}, \varepsilon_{1}=2.25(\tan \delta=0.001)$, $\varepsilon_{2 a}=4.40(\tan \delta=0.02), \quad \varepsilon_{2 b}=(1.2-j 103.5) \times 10^{6}$
(Copper), $\mu_{1}=\mu_{2 a}=\mu_{2 b}=1$; where $(\varepsilon, \mu)_{2 a, 2 b}$ represents the $\varepsilon$ and $\mu$ of the different inclusions. A 6-by-6 array was used in the plane of the infinite/symmetry boundaries, that is, the yz-plane, and the structure had 11 layers, implying a thickness of $3.3 \mathrm{~mm}$ in the direction of propagation of the plane wave - the $x$-axis. Perfect Electric and Perfect Magnetic Conductor (PEC and PMC) boundary conditions are used along the $z$ - and $y$-axes respectively. They act as symmetry planes to create an infinite structure along those boundaries. Absorbing boundary conditions are used in the $x$-axis to minimize reflections as the wave travels. An indepth description of these boundaries is given in [15]. Fig. 5 shows the $\varepsilon_{e f f}$ and $\mu_{\text {eff }}$ of the structures in Fig. 4 and includes the cases when the spheres are either all dielectric or all metallic.

As shown in Fig. 5, as the volume fraction of the metallic inclusions increases, the $\varepsilon_{\text {eff }}$ increases while the $\mu_{e f f}$ decreases, as expected. Table 1 gives a summary of the important properties of these heterogeneous media, where $p_{d}$ and $p_{m}$ are the volume fractions of the dielectric and metallic inclusions respectively. Even though spherical inclusions are used, the orientation of the rows of metallic/dielectric spheres has been shown to have an effect on the $\varepsilon_{e f f}$. As shown, the extracted values of $\varepsilon_{e f f}$ from the simulation-inversion process agrees well with the analytical results from [9] as shown in equation (6):

$$
\varepsilon_{e f f}=\varepsilon_{1}+3 \varepsilon_{1} \frac{\sum_{k=2}^{K} p_{k} \frac{\varepsilon_{k}-\varepsilon_{1}}{\varepsilon_{k}+2 \varepsilon_{1}}}{1-\sum_{k=2}^{K} p_{k} \frac{\varepsilon_{k}-\varepsilon_{1}}{\varepsilon_{k}+2 \varepsilon_{1}}}
$$

where $k$ represents the different inclusions. The extracted values are the average values over the frequency range examined. There are no results for the $\mu_{\text {eff }}$ from [9] as $\mu_{e f f}=1$, in all cases as explained in Section 3. With increased volume fractions of the metallic inclusions, the $\varepsilon_{\text {eff }}$ will further increase while the $\mu_{\text {eff }}$ will decrease.

Table 1: Summary of simulated results

\begin{tabular}{|l|c|c|c|c|c|}
\hline & $p_{d}$ & $p_{m}$ & $\varepsilon_{\text {eff }}$ & $\varepsilon_{\text {eff }}[9]$ & $\mu_{\text {eff }}$ \\
\hline y-oriented & 0.071 & 0.085 & 3.04 & 3.01 & 0.89 \\
\hline$x$-oriented & 0.078 & 0.078 & 2.97 & 2.97 & 0.89 \\
\hline Alternate & 0.078 & 0.078 & 2.98 & 2.97 & 0.90 \\
\hline All-dielectric & 0.155 & - & 2.51 & 2.51 & 1.00 \\
\hline All-metallic & - & 0.155 & 3.54 & 3.49 & 0.79 \\
\hline
\end{tabular}

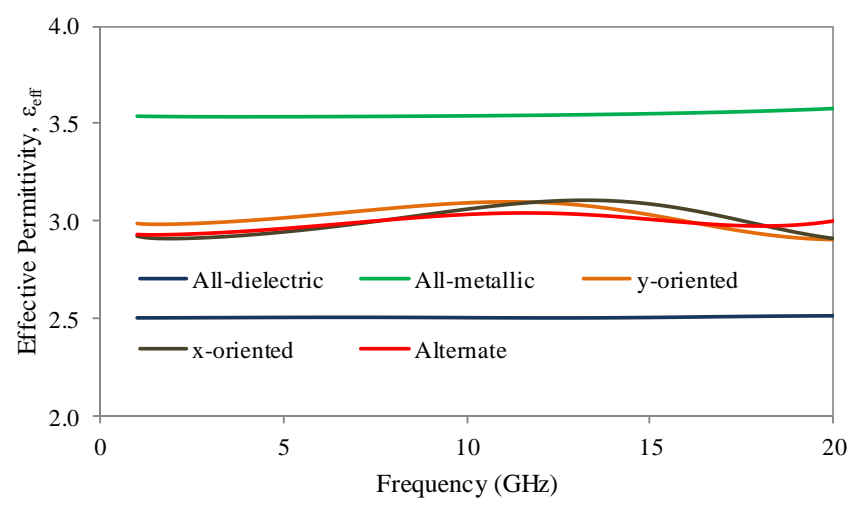

(a) 


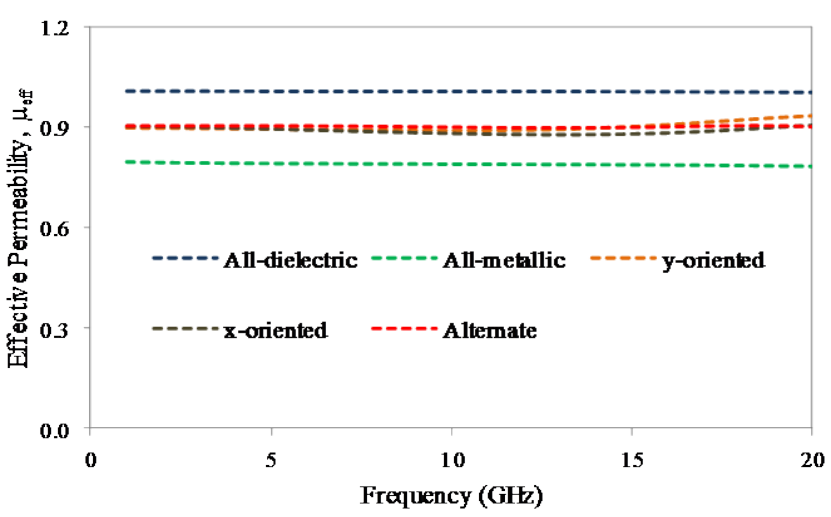

(b)

Figure 5: Variation of (a) $\varepsilon_{\text {eff }}$ (continuous) and (b) $\mu_{\text {eff }}$ (dashed) with frequency for different heterogeneous mixtures

\section{Patch Antenna}

As mentioned earlier, heterogeneous mixtures can be used as antenna substrates. In this section, the geometry and simulated results of simple example are given. Fig. 6 shows the geometry of a simple inset-fed patch antenna on a heterogeneous medium with dielectric cubic inclusions. Data used: patch dimensions: $24 \mathrm{~mm}$ by $27.76 \mathrm{~mm}$, transmission line width $=3.12 \mathrm{~mm}$, inset feed depth $=8 \mathrm{~mm}$, cube length, $l=0.5 \mathrm{~mm}$, spacing, $s=0.75 \mathrm{~mm}$, thickness of the substrate, $d_{s}=1.5 \mathrm{~mm}, \varepsilon_{1}=2.08(\tan \delta=0.0004), \varepsilon_{2}=$ $12.94(\tan \delta=0.006), \mu_{1}=\mu_{2}=1$. The $\varepsilon_{\text {eff }}$ for the heterogeneous medium is 3.55 ( $\tan \delta=0.0016$ ) obtained from equation (1), and is used as the permittivity for the homogeneous equivalent. The return loss, $S_{11}$, for these two structures are shown in Fig. 7. The results show good agreement with the resonant frequency of the heterogeneous substrate $(3.21 \mathrm{GHz})$ being slightly lower than that of the homogenous equivalent (3.25 GHz). Previous work by the authors in [22], [23] have shown this to be the case for spherical and metallic inclusions. The radiation efficiencies are $93.52 \%$ and $92.82 \%$ for the homogenous and heterogeneous respectively which again show good agreement. These good agreements provide further confidence that the canonical equations accurately predict the effective EM properties of these heterogeneous media. This shows that the match is maintained and reasonable efficiencies are possible.

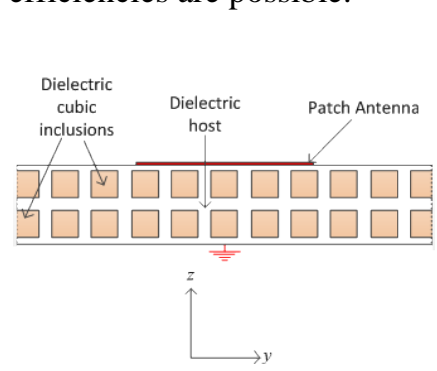

(a)

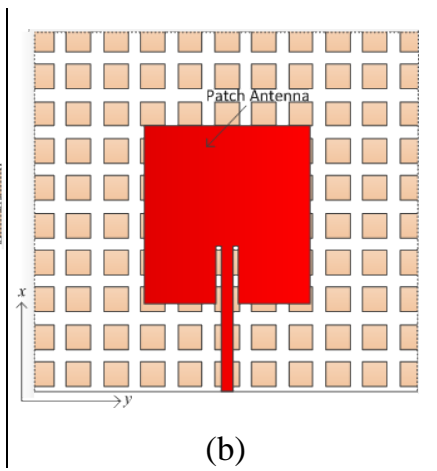

(b)
Figure 6: (a) Side view and (b) top view of inset-fed patch antenna on a heterogeneous substrate (not drawn to scale)

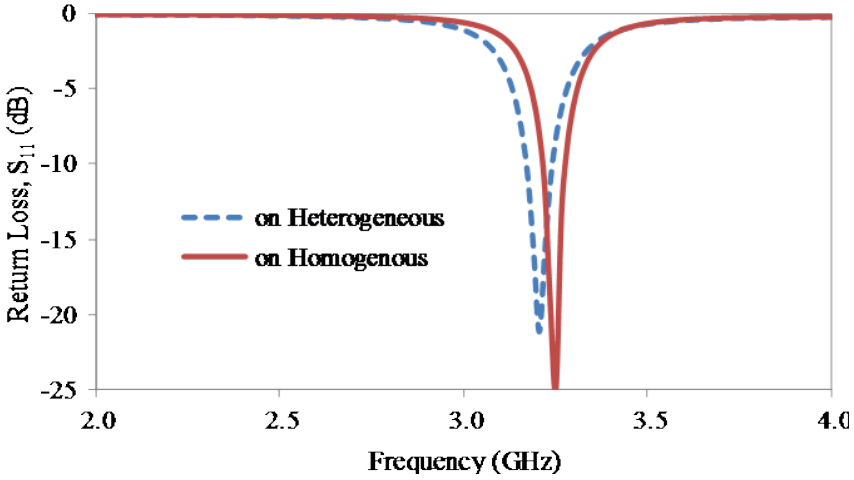

Figure 7: Simulated $S_{11}$ of same sized patch on a homogenous (continuous) and heterogeneous (dashed) substrates with dielectric cubic inclusions

\section{Conclusions}

Canonical equations from different authors have been compared with each other and with simulated results. Even though the equations appear to be different, on closer inspection and under certain assumptions, they can be shown to be quite similar to each other. Heterogeneous mixtures with more than one type of inclusion have been simulated with the results showing good agreement with the equivalent canonical equation. An example patch antenna on a heterogeneous substrate with dielectric cubic inclusions have been simulated and shown to have good agreement with when the patch antenna is on its homogenous equivalent substrate.

\section{Acknowledgements}

This work was supported by EPSRC under Grant No. EP/I01490X/1.

\section{References}

[1] M. I. Kitra, C. J. Panagamuwa, P. McEvoy, J. (Yiannis) C. Vardaxoglou, and J. R. James, "Low SAR ferrite handset antenna design," IEEE Transactions on Antennas and Propagation, vol. 55, no. 4, pp. 1155-1164, Apr. 2007.

[2] G. Kiziltas, D. Psychoudakis, J. L. Volakis, and N. Kikuchi, “Topology design optimization of dielectric substrates for bandwidth improvement of a patch antenna," IEEE Transactions on Antennas and Propagation, vol. 51, no. 10, pp. 2732-2743, Oct. 2003.

[3] C.-C. Chen and J. L. Volakis, "Bandwidth broadening of patch antennas using nonuniform substrates," Microwave and Optical Technology Letters, vol. 47, no. 5, pp. 421-423, Dec. 2005.

[4] D. Psychoudakis, Y.-H. Koh, J. L. Volakis, and J. H. Halloran, "Design method for aperture-coupled microstrip patch antennas on textured dielectric substrates," IEEE Transactions on Antennas and Propagation, vol. 52, no. 10, pp. 2763-2765, Oct. 2004.

[5] D. Psychoudakis, J. L. Volakis, Z. N. Wing, S. K. Pillai, and J. W. Halloran, "Enhancing UHF antenna 
functionality through dielectric inclusions and texturization," IEEE Transactions on Antennas and Propagation, vol. 54, no. 2, pp. 317-329, Feb. 2006.

[6] Lord Rayleigh, "On the influence of obstacles arranged in rectangular order upon the properties of a medium,” Philosophical Magazine, vol. 34, pp. 481-502, 1892.

[7] L. Lewin, "The electrical constants of a material loaded with spherical particles,” IEE-Part III: Radio Comm. Eng., vol. 94, no. 27, pp. 65-68, 1947.

[8] X. Cai, R. Zhu, and G. Hu, "Experimental study for metamaterials based on dielectric resonators and wire frame,” Metamaterials, vol. 2, no. 4, pp. 220226, 2008.

[9] A. Sihvola, Electromagnetic Mixing Formulas and Applications. London: IET, 1999.

[10] R. E. Collin, Field Theory of Guided Waves. New York: IEEE Press, 1991.

[11] W. T. Doyle, "The Clausius-Mossotti problem for cubic arrays of spheres," Journal of Applied Physics, vol. 49, no. 2, pp. 795-797, 1978.

[12] W. T. Doyle, "Optical properties of a suspension of metal spheres,” Physical Review B, vol. 39, no. 14, pp. 9852-9858, 1989.

[13] I. A. Kolmakov, L. Jylha, S. A. Tretyakov, and S. Maslovki, "Lattice of dielectric particles with double negative response,” in 28th Gen. Ass. Int. Union Radio Sci. (URSI), 2005.

[14] C. C. Njoku, W. G. Whittow, and J. C. Vardaxoglou, "Comparative study of nanomaterials' effective properties using canonical formations,” in Loughborough Antennas \& Propagation Conference (LAPC), 2010, pp. 413-416.

[15] C. C. Njoku, W. G. Whittow, and Y. C. Vardaxoglou, "Simulation methodology for synthesis of antenna substrates with micro-scale inclusions,” IEEE Transactions on Antennas and Propagation, vol. 60, no. 5, pp. 2194-2202, 2012.

[16] C. C. Njoku, W. G. Whittow, and Y. C. Vardaxoglou, "Effective permittivity of heterogeneous substrates with cubes in a 3-D Lattice," IEEE Antennas and Wireless Propagation Letters (Special Issue on Metamaterials), vol. 10, pp. 1480-1483, 2011.

[17] R. Asmatulu, B. Geist, W. B. Spillman, and R. O. Claus, "Dielectric constant and breakdown field studies of electrostatic self-assembled materials," Smart Materials and Structures, vol. 14, no. 6, pp. 1493-1500, Dec. 2005.

[18] C. L. Holloway, E. F. Kuester, J. Baker-Jarvis, and P. Kabos, “A double negative (DNG) composite medium composed of magnetodielectric spherical particles embedded in a matrix," Antennas and Propagation, IEEE Transactions on, vol. 51, no. 10, pp. 2596-2603, 2003.

[19] J. C. Vardaxoglou, "Optical switching of frequency selective surface bandpass response,” Electronics Letters, vol. 32, no. 25, pp. 2345-2346, 1996.
[20] I. Awai, O. Mizue, and A. K. Saha, “Artificial dielectric resonator made of spherical metal particles," IEICE Transactions on Electronics, vol. E92-C, no. 1, pp. 72-76, 2009.

[21] W. M. Merrill, R. E. Diaz, M. M. LoRe, M. C. Squires, and N. G. Alexopoulos, "Effective medium theories for artificial materials composed of multiple sizes of spherical inclusions in a host continuum," IEEE Transactions on Antennas and Propagation, vol. 47, no. 1, pp. 142-148, 1999.

[22] C. C. Njoku, W. G. Whittow, and Y. C. Vardaxoglou, "Designing microwave patch antennas using heterogeneous substrates," in European Conference on Antennas and Propagation (EuCAP), 2012, pp. 886-888.

[23] W. G. Whittow, C. C. Njoku, and J. C. Vardaxoglou, "Designing multi-band and high bandwidth antennas with heterogeneous substrates," in IEEE Tropical Conference on Antennas and Propagation in Wireless Communications (APWC), 2012, pp. 748751. 